Revista Calidad en la Educación Superior

Programa de Autoevaluación Académica

Universidad Estatal a Distancia

ISSN 1659-4703

Costa Rica

revistacalidad@uned.ac.cr

\title{
Gestión en la Administración del Aprendizaje y Herramientas en la Sistematización de Experiencias
}

Management in the Learning Management and Tools Systematization of Experiences

Rosita Ulate Sánchez ${ }^{1}$

rulate@uned.ac.cr

Universidad Estatal a Distancia, Costa Rica

\author{
Volumen 1, Número 1 \\ Mayo 2010 \\ pp. $81-96$
}

Recibido: abril, 2010

Aprobado: mayo, 2010

\footnotetext{
${ }^{1}$ Graduada en Administración de Empresas con una Maestría con Énfasis en Mercadeo en la Fundación de Estudios de Pos grado de la Universidad de Costa Rica
} 
Gestión en la Administración del Aprendizaje y Herramientas en la Sistematización de Experiencias Rosita Ulate Sánchez

\section{Resumen}

La adaptación de las organizaciones educativas es uno de los principales retos en el Siglo XXI ante los avances tecnológicos en los procesos de la información y la comunicación. Innovaciones que se convierten en herramientas en la generación del conocimiento organizacional y de aquellas áreas del saber en las que se apliquen. De allí la importancia de recuperar las experiencias de la gestión en el proceso de enseñanza y aprendizaje a través del adecuado procesamiento de la información existente. Para ello existen una serie de herramientas automatizadas que permiten generar servicios de calidad a los actores sociales de una comunidad universitaria. Sin embargo su aprovechamiento oportuno depende de las toma de decisiones administrativas de las instituciones educativas.

Palabras claves: Gestión administrativa; sistematización de experiencias, herramientas en el aprendizaje.

\section{Summary}

Educational organizations adaptation is one of the main challenges in the $\mathrm{XXI}$ century to the technological advances in information processes and communication. Innovations become tools in the creation of organizational knowledge and knowledge of those areas in which they apply. Hence the importance of recovering the experience of handling the teaching and learning process, through the proper managing of existing information. This is a series of automated tools that allow you to generate high-quality services to stakeholders of a university community. However their appropriate use depends on the management decisions of educational institutions.

Keywords: Administrative management, systematization of experiences, tools for learning. 
Gestión en la Administración del Aprendizaje y Herramientas en la Sistematización de Experiencias Rosita Ulate Sánchez

\section{Introducción}

El acceso a determinados recursos en la historia de la civilización ha dado una ventaja competitiva a quién lo posee. La teoría de Charles Darwin deja claro que las especies que sobreviven no son las más fuertes o inteligentes, sino las que logran adaptarse al ambiente. Es así como que en la era agrícola quien tenía acceso a la tierra era el beneficiado; en la era industrial quién poseía capital tenía una ventaja sobre los que no; en la era de la información, quién tiene acceso a ella tendrá más oportunidades sobre los que no; así como quienes tienen acceso a la tecnologías de la información y la comunicación serán parte de los beneficiados en la era del conocimiento (Gómez, Pérez y Curbelo (2005). No porque la información sea conocimiento, sino porque a través del acceso a ella se podrá generar modificaciones y nuevos modelos mentales que pueden convertirse en un saber. La educación desde las grandes innovaciones tecnológicas busca hacer uso de ella como instrumento para que la información pueda ser consultada a través de motores de búsqueda, almacenamiento y consulta (Barberá, 2009). La misma autora indica que es la calidad de la propuesta tecnológica y el uso que se le dé a la información disponible, lo que indicará si se genera o no un aprendizaje, un conocimiento en el proceso de enseñanza y aprendizaje.

La transmisión de conocimientos, de experiencias de personas y organizaciones es un reto que promueve la utilización de herramientas en la gestión del conocimiento al resto de la sociedad.

\section{Información y conocimiento}

Es necesario establecer la diferencia entre información y conocimiento; información es "el conjunto de datos relacionados e interpretados en un contexto específico (...) para obtener conocimiento” (Gómez, Pérez \& Curbelo, 2005, p.38). Ahora bien, conocimiento, desde la perspectiva de Davenport y Prusak (mencionado 
Gestión en la Administración del Aprendizaje y Herramientas en la Sistematización de Experiencias Rosita Ulate Sánchez

por Gómez et al. 2005) será "una mezcla de experiencias, valores, información y saber hacer que sirve como marco para la incorporación de nuevas experiencias e información, y es útil para la acción". De tal forma que el conocimiento se origina y aplica en la mente de los conocedores; mismo que se expresa en las organizaciones por medio de documentos, almacenes de datos y en las rutinas organizativas, procesos, prácticas y normas.

\section{Gestión del conocimiento}

De acuerdo con Goméz et.al (2005) la gestión del conocimiento se puede definir como "el proceso sistemático que utiliza el conocimiento individual y colectivo de la organización orientada a potenciar las competencias organizacionales y la generación de valor en pro de obtener ventajas competitivas sostenibles" (p.41). En este sentido la gestión del conocimiento es una poderosa herramienta para la administración, producción y distribución del mismo y utilizable como una ventaja competitiva, sea personal u organizacional.

Berrocal y Pereda (2004) mencionan tres pilares en la gestión del conocimiento en una organización: "una adecuada cultura y estilo de dirección, un sistema de gestión de persona por competencias y un soporte tecnológico que facilite el acceso y distribución del conocimiento" (p.1); lo anterior se limita a dos áreas: la técnica (gestión de la información y tecnología según Gómez, et. al (2005) y la humana (Berrocal y Pereda (2004). Áreas que permiten la distribución y transferencia de un conocimiento generado a través de experiencias y vivencias.

Las herramientas de la gestión del conocimiento a través del establecimiento de un sistema facilita la renovación de estructuras mentales a lo interno de las organizaciones. En las organizaciones se genera un conocimiento innato en la resolución de problemas y en la toma de decisiones, de tal forma que las personas logran adquirir a través de las experiencias vividas información valiosa y conocimiento, 
Gestión en la Administración del Aprendizaje y Herramientas en la Sistematización de Experiencias Rosita Ulate Sánchez

el cual es conocido en el idioma inglés como el "know how" considerado como parte del capital intelectual. Razón por la cual las organizaciones promueven la recopilación de ese conocimiento para ser trasladado a otros individuos y organizaciones.

La gestión del conocimiento de una organización se enmarca al "obtenido en un momento y lugar determinados, para permitir trasladarlo y aplicarlo a otro lugar 0 momento, para obtener los resultados esperados en menos tiempo y con menos errores" (Aledo, 1998 citado por Berrocal \& Pereda, 2004). Se tratará entonces de no perder ese conocimiento creado, razón por la que las organizaciones buscan soluciones para gestionarlo, compartirlo, renovarlo y ampliarlo de una forma articulada y táctica para que se convierta en un verdadero capital intelectual.

De acuerdo con Benavides y Quintana (2003) la gestión del conocimiento es un sistema socio-técnico en el que será necesario la tecnología, un proceso organizativo, capital intelectual y social en el que según Carayanis (1998) mencionado en Benavides y Quintana (2003) la tecnología de la información es una infraestructura que permite la creación de un valor agregado del quehacer y del saber hacer de una organización.

\section{Perspectivas en las herramientas del aprendizaje}

Cifras de Appleberry (s.f.) citado por Guadamuz (2006) indica que el conocimiento con una base disciplinar y expuesto a nivel internacional, contado desde el inicio de la era cristiana, demoró 1759 años en duplicarse por primera vez; para que luego su volumen llegase a duplicarse cada 150 años, luego cada 50 años, hasta llegar a cada cinco años en el siglo XXI; proyectando que para el año 2020 se duplicará cada 73 días. De tal forma que el conocimiento cambia con nuevas características; con un crecimiento acelerado y de alta complejidad en el área de la educación. Según Gudamuz (2006) es una nueva era científica, la era de las 
Gestión en la Administración del Aprendizaje y Herramientas en la Sistematización de Experiencias Rosita Ulate Sánchez

posibilidades y probabilidades en donde se educará para el cambio y la incertidumbre, generando un nuevo conocimiento.

Charles Handy (1992), gurú de la administración, ha indicado que "un mundo cambiante exige nuevas ideas. Pensar lo imposible es una forma de mover la rueda del aprendizaje" (citado por Soto \& Sauquet, 2006, p.152); desde esta perspectiva se ha desarrollado toda una teoría administrativa que expone la importancia de que las organizaciones y las personas aprendan como un factor de éxito organizacional o personal. Este enfoque promueve la cultura del auto aprendizaje basado en un modelo de aprender a aprender, llamado en el ambiente empresarial como las organizaciones que aprenden muy de la mano con el enfoque de mejora continua.

Según las perspectivas de la Federación para el Aprendizaje (2003) en un futuro cercano, hacia el año 2020, los sistemas de aprendizaje deberán cambiar de uno que es "empujado por el maestro" a uno" jalado por el estudiante" en donde serán los estudiantes los que usen la tecnología como buscadores o repositorios de información. Es decir que los sistemas deberán brindar la posibilidad de que el estudiante tenga acceso a información de múltiples fuentes y de un conocimiento compartido. La misma federación indica que las herramientas futuras:

incluirán sistemas para: evaluación continua de los progresos del aprendiz hacia el alcance de la competencia en áreas críticas; respuestas individuales, contextualizadas y oportunas a preguntas que se plantearán a sistemas automáticos, a tutores reales y a expertos; construcción rápida de redes de aprendices y maestros; monitoreo del nivel de interés, ansiedad y motivación del usuario; adaptación a aprendices específicos (idioma de instrucción, antecedentes culturales, habilidades de aprendizaje); y sistemas para construir simulaciones que reflejen el estado del arte en ciencias, ingeniería y otros campos (p.4).

Ahora bien, las transformaciones que se visualizan en los sistemas de gestión del conocimiento exigirán "cambios dramáticos en las estructuras organizacionales y en los sistemas administrativos de las instituciones educativas y de los servicios de entrenamiento" (p.10).

\section{Administración de la gestión del conocimiento}


Gestión en la Administración del Aprendizaje y Herramientas en la Sistematización de Experiencias Rosita Ulate Sánchez

La administración de la gestión del conocimiento implica su organización con al menos los siguientes propósitos: a) expandir o difundir el conocimiento a toda la organización; b) mejorar la accesibilidad a la información y el conocimiento por parte de los funcionarios; c) facilitar el proceso de colaboración e investigación; d) fomentar la innovación y e) comparte las mejores prácticas a quién necesite aprender (Gómez et.al., 2005, pp. 43-44).

El enfoque que debería prevalecer en una buena gestión del conocimiento en una organización es comprender que son las personas los depositarios y gestores del conocimiento. Desde esta perspectiva se deberá aprovechar las experiencias y las competencias desarrolladas por el personal docente en una institución educativa. Para ello Gómez, et. al (2005) sugiere por ejemplo:

a) Promover a los funcionarios de una organización como motores de la innovación;

b) Crear una cultura de mejora y aprendizaje continua;

c) Convertir el conocimiento personal en competencias organizacionales y en ventajas competitivas.

d) Capitalizar el conocimiento creado y lograr su retorno en formación del personal;

e) Buscar estrategias de experimentación y solución a los problemas;

f) Aprender más rápidamente y sacar provecho de la experiencia colectiva;

g) Transferir el conocimiento adquirido a todos en la organización.

\section{Sistematización de experiencias}

Se define como "aquella interpretación crítica de una o varias experiencias que, a partir del ordenamiento y reconstrucción, descubre o explica la lógica del proceso vivido en ellas" (Jara 2006, p.9). La sistematización educativa es concebida como una 
Gestión en la Administración del Aprendizaje y Herramientas en la Sistematización de Experiencias Rosita Ulate Sánchez

herramienta de lectura y producción colectiva de una realidad específica, más allá de un procedimiento de etapas y técnicas preestablecidas (Messina, 2004).

De acuerdo con Estrada (2005), la sistematización nace como una práctica de la educación no formal, desde la iniciativa de organizaciones no gubernamentales para recolectar el conocimiento popular en la década de los setenta. A partir de la década de los ochenta y principios de los noventa, se contextualiza como un acierto para la creación de unidades didácticas en el trabajo social, lo cual dio como resultado que la sistematización se incorporar a la educación formal.

Para Jara (1994) la sistematización es un proceso en el que se adquiere conocimiento al rescatar, descubrir, ordenar, jerarquizar, interpretar y reflexionar sobre experiencias dinámicas. Es la articulación creativa, modificación y mejora del saber práctico y teórico en la cual se da la participación de los actores sociales.

Dentro de las características de la sistematización ofrecidas por Jara (1994) se pueden mencionar: a) la producción de un nuevo conocimiento (la objetividad de lo vivido), b) el ordenamiento de los conocimientos y de las percepciones, c) la creación de un espacio para compartir, confrontar y discutir, y d) el mantenimiento de una memoria histórica y e) la facilitación del acceso a este tipo de información.

Castillo (2002), Senlle y Gutiérrez (2005) se refieren a la sistematización en la educación como una metodología para registrar los procesos realizados. Los mismos autores afirman que ésta es de suma importancia dentro de la autoevaluación educativa, pues permite detectar fortalezas existentes y oportunidades de mejora.

Desde el punto de vista educativo específicamente, se encuentran pocos conceptos que precisen la sistematización; pero uno de ellos es el expuesto por Messina (2004), quien la define como una herramienta de lectura y producción colectiva de una realidad específica, más allá de un procedimiento de etapas y técnicas preestablecidas. 
Gestión en la Administración del Aprendizaje y Herramientas en la Sistematización de Experiencias Rosita Ulate Sánchez

lovanovich (2007) explica la sistematización como una forma específica de investigación que permite la recuperación posterior a la práctica de "los saberes y conocimientos que han sido eficaces para operar sobre la realidad" (p.1). El mismo autor indica que en la "práctica docente (la sistematización) es una búsqueda y como tal, se propone sacar a la luz la teoría explícita o implícita de la práctica" (p.2). Menciona, además, la importancia de un marco de métodos, técnicas e instrumentos utilizados en las ciencias sociales.

Por lo anteriormente expuesto se logra inferir que las experiencias y vivencias en una organización educativa tienen una gran posibilidad de formar parte del conocimiento como producto de su capital intelectual, el cual puede ser transferido y por lo tanto accesado, estudiado, analizado para futuras situaciones en las que se busca una nueva construcción, sea de conformación de teorías o bien en la aplicación directa de resolución de problemas y toma de decisiones educativas.

\section{Gestión del conocimiento tácito y explicito por medio de la sistematización de}

\section{experiencias}

Queda claro cómo la sistematización es una de las estrategias para recolectar en algún medio impreso o audio visual, la riqueza de experiencias que en el campo educativo quedan muchas veces ocultas al no formar parte de las exposiciones a través de las publicaciones formales en la comunidad educativa.

Varias iniciativas en la sistematización de experiencias muestran como se puede lograr recolectar, ordenar y gestionar el conocimiento tácito (que no se encuentra codificado y dentro de una categoría de experiencias tales como de trabajo, emocionales, vivenciales, de know how, de habilidades, de creencias) ó conocimiento explicito (que se encuentra de alguna forma codificado y posible de transferir en algún sistema de lenguaje formal, como en documentos, mensajes, presentaciones, 
Gestión en la Administración del Aprendizaje y Herramientas en la Sistematización de Experiencias Rosita Ulate Sánchez

diseños, especificaciones, procedimientos, simulaciones, entre otros) (Gómez, et.al, 2005).

Existen varias muestras de la existencia de la trayectoria social en la sistematización; entre éstas se cuenta con el Programa Latinoamericano de Apoyo a la Sistematización (CEAAL) con la biblioteca virtual de sistematización de experiencias (CEAAL, 2009). Otro de los esfuerzos en sistematización es La Red Innovemos, con objetivos dentro del Convenio Andrés Bello 2003-2012 (Políticas, estrategias y consensos de acción en ciencia y tecnología), para "construir de forma permanente un marco de referencia compartido sobre la teoría y práctica del cambio y la innovación educativa en la región" (2004, p.65), y produciendo, a la vez, conocimientos teóricos y prácticos a través de los procesos de investigación, sistematización y evaluación de las innovaciones. También se observa una iniciativa de sistematización de experiencias realizada por Roca y Prat (Prat \& Veyoda, 2008), en la que se recogen en una base de datos todas las aplicaciones informáticas de materiales didácticos multimedia producidos para uso docente universitario en la carrera de arquitectura de la Universidad Nacional del Noreste de Argentina, con el fin de desarrollar un producto final que integre lo mejor de los esfuerzos anteriores.

\section{Herramientas de administración del aprendizaje (LMS y LCMS)}

Los sistemas de gestión de aprendizaje (LMS) conocidos también como plataformas o campus virtuales son una aplicación de software que posibilita la creación de un entorno para la enseñanza y el aprendizaje en línea, de tal forma que administra el proceso y todas sus actividades, sea contenidos instruccionales, identifica, supervisa y evalúa, almacena, calendariza, en algunas ocasiones también regula la matrícula y la logística de los cursos (Brandon Hall, 2008 en Greenberg, 2008; Watson, 2007). De acuerdo con ARI: Soluciones E-learning, los LMS es una 
Gestión en la Administración del Aprendizaje y Herramientas en la Sistematización de Experiencias Rosita Ulate Sánchez

aplicación residente en un servidor de páginas web en la que se desarrollan las acciones formativas (...) es el lugar donde alumnos, tutores, profesores o coordinadores se conectan a través de internet para descargar contenidos, ver el programa de asignatura, enviar un correo al profesor, charlar con los otros compañeros, debatir en un foro, participar en una tutoría (p.1)

Algunas de las alternativas en idioma español son: Online Learning Solution (SAP); Sistemas Avanzados de Tecnología (SATEC); Oracle; VideoChat, Kedros, Tralcom: Classroom coordinator, Competir: Web Campus.

Por otro lado los sistemas de gestión de contenidos de aprendizaje (LCMS) igualmente administran todas las formas de aprendizaje que ofrezca una organización, sin embargo su enfoque esta en el contenido del aprendizaje en línea por medio de objetos de aprendizaje; además crean, almacenan, ensamblan y entrega de forma personalizada. ARI: Soluciones E-learning (s.f.) indica que este tipo de herramientas como el LCMS "es un sistema independiente o integrado con el LMS (Plataforma), que gestiona y administra los contenidos de aprendizaje. Una vez que los contenidos están en este sistema ya pueden ser combinados, asignados a distintos cursos, descargarlos desde el archivador electrónico" (p.1).

Greenberg (2008) indica que incluye tres componentes: un objetivo de rendimiento, el contenido de aprendizaje y alguna forma de evaluación.

Tanto el LMS como LCMS son complementarios, pero su mayor diferencia radica según Greenberg (2008) es que los LCMS en lugar de desarrollar los cursos y su adaptación a audiencias generalizadas, los diseñadores de la instrucción crean bloques de contenido reutilizables y los ponen a disposición de desarrolladores de todo un curso de la organización.

\section{Tipos de herramientas para la administración de la experiencia}

Con el propósito de conocer el tipo de herramientas y su aplicación en la administración de la experiencia, se presenta una serie de posibilidades, algunas en la 
Gestión en la Administración del Aprendizaje y Herramientas en la Sistematización de Experiencias Rosita Ulate Sánchez

práctica actualmente, todas con un breve comentario y la dirección electrónica para conocer un poco más de este tipo de iniciativas.

Bases de datos de mejores práctica. En este tipo de herramienta se puede encontrar ubicada la Iniciativa ADRS, desarrollada para dar a conocer experiencias y contribuciones dentro de un proceso voluntario de compromisos orientados a las comunidades en el desarrollo sostenible. Para ello garantiza en base de datos recursos, experiencia, pericias, conocimientos y tecnologías para fortalecer las capacidades, iniciativas e innovaciones de los agricultores, pescadores artesanales, pastores y otras personas que viven en el campo, con miras a lograr. Lo que busca la iniciativa ADRS es reunir pericias, conocimientos y tecnologías con las exigencias de las comunidades rurales y las partes interesadas menos favorecidas. Mediante el incremento de las lecciones aprendidas, los esfuerzos exitosos y los diversos enfoques, la Iniciativa ADRS ayudará a ampliar el acceso y el uso de los recursos existentes, así como a obtener mayores beneficios de ellos. Para ello ha concretado bases de datos sobre buenas prácticas con una evaluación previa de cada una de ellas, de tal manera que expone las ventajas y fortalezas de cada base de datos recomendada; la dirección es http://www.fao.org/sard/es/init/1574/1507/index.html.

Bases de datos de lecciones aprendidas. La creación del Programa de Mejores Prácticas y Liderazgo Local de UN-Habitat es creada en el Foro Iberoamericano y del Caribe sobre mejores prácticas, consiste en una red de instituciones que busca beneficiarse de las experiencias y lecciones aprendidas como vehículo de aprendizaje y aplicabilidad en diferentes áreas de especialidad. El propósito es buscar un mejor uso, evaluación, entendimiento y aprovechamiento de las mejores prácticas en Iberoamerica y del Caribe como instrumentos de participación local y de la sociedad civil en los procesos de mejora en el nivel de vida. Para ello ha creado una base de 
Gestión en la Administración del Aprendizaje y Herramientas en la Sistematización de Experiencias Rosita Ulate Sánchez

datos

disponible

en

la

dirección

electrónica

http://habitat.aq.upm.es/busqueda/bestpr.html. En la cual se ha identificado y analizado las mejores prácticas de la región, para promover su transferencia.

Manuales digitales. Es un tipo de herramienta con la cual se puede crear material de apoyo con información pertinente, generalmente utilizada por los comunicadores. Los sistemas de administración de contenidos (CMS) con parte de esta categoría, entre ellos los blogs y las wikis (http://fcomudep.net/textos/manual herramientas digitales.pdf). Algunos ejemplos en el campo del periodismo pueden ser observados en un curso de este tema: http://www.centroperiodismodigital.org/manuales.

Bases de datos de preguntas frecuentes (FAQ). Son herramientas generadoras de aplicaciones que puedan crear fácilmente una lista de preguntas frecuentes, a través de una zona de administración poderosa que incluye una navegación dinámica; en versiones más novedosas se encuentras aplicaciones para el envío de mensajes de texto telefónicos. Algunos de los software para estas aplicaciones pueden ser visualizadas en la dirección electrónica:

http://asp.astalaweb.com/FAQS/1 FAQS.asp

Herramientas help desk. Los HelpDesk contienen una base de datos de conocimiento que guarda todas las soluciones ofrecidas previamente y que pueden ser accesibles por el personal, el número de respuestas va aumentando con el tiempo, de forma que

se reduce el tiempo de gestión de las solicitudes ya que no es necesario contestar una misma pregunta dos veces. Todas las soluciones se guardan en una única base de datos, la cual es utilizada por el personal de soporte para buscar una solución cuando se registra una solicitud nueva, o mientras se está gestionando la solicitud. De esta forma, se puede saber si ya existe una solución documentada sobre una solicitud similar (Artologik, s.f.). 
Gestión en la Administración del Aprendizaje y Herramientas en la Sistematización de Experiencias Rosita Ulate Sánchez

\section{Conclusiones}

Las diferentes civilizaciones de la historia han desarrollado un saber autóctono que ha sido enseñado a través de metodologías de aprender haciendo. Esta forma de aprender es desde siglos atrás un acompañante en la generación del conocimiento humano propia de la acción colectiva. La sistematización de experiencias permite procesos de reflexión en los cuales se dan oportunidades de mejoramiento de procesos educativos. Por otro lado también la sistematización es una práctica para la reconstrucción histórica de los procesos, así como de la organización de la información producida, sea documental, fotográfica, vivencial, entre otras.

En lo interno de las organizaciones educativas se dan diariamente una serie de prácticas en la resolución de problemas administrativos, didácticos, técnicos, pedagógicos que muy pocas veces son registrados y dispuestos para consulta. Difícilmente se aprovecha los resultados de asignaciones, trabajos de campo e investigaciones realizadas por los estudiantes que ameriten ser comunicados, sean estos producidos en formas tradicionales o en LMS y LCMS, en cuyas plataformas no se ha observado un tipo de apartado para sistematizar o publicar las experiencias aprendidas.

El conocimiento producto de las experiencias y lecciones aprendidas es una competencia silenciosa que es invisibilizada por la falta de una cultura de sistematización, sobre todo en una época de revolución tecnológica que permite a través de varias herramientas registrar, organizar, comunicar y transferir ese conocimiento a otros individuos y organizaciones.

El conocimiento es generado por el ser humano, cada vez en una producción exponencial de crecimiento, pero así también de acelerado son las herramientas que 
Gestión en la Administración del Aprendizaje y Herramientas en la Sistematización de Experiencias Rosita Ulate Sánchez

permite la tecnología. ¿Por qué no devolver a la sociedad el conocimiento generado en los procesos de enseñanza y aprendizaje de las instituciones de educación superior?

\section{Referencias}

Ari: Soluciones E-learning. (s.f.). El E-learning y su terminología. Recuperado el 29 de marzo 2010 en http://elearning.ari.es/articulos/el elearning y su terminologia.html

Artologik. (s.f.). Help Desk. Recuperado el 28 de marzo 2010 en http://www.artologik.com/es/HelpDesk/Acerca-del-Programa/Base-de-datos-yFAQ.aspx http://www.artologik.com/es/HelpDesk/Acerca-del-Programa/Basede-datos-y-FAQ.aspx

Barberá, E., Mauri, T. \& Onrubia, J. (Cords.). Aguado, G. Badia. A., C. C., Colomina, R., Engel, A., Espasa A., Lafuente M., Narango, M. \& Rochera, J. (2008). Cómo valorar la calidad de la Enseñanza Basada en las TIC. Pautas e instrumentos de análisis. Madrid, España: Imprimeix.

Benavides, C. \& Quintana, C. (2003). Gestión del conocimiento y calidad total. Madrid, España: Ediciones Díaz de Santos S.A.

Berrocal, F. \& Pereda, S. (2004). Generación de conocimiento y gestión de recursos humanos. (84-89). En Cambiar con la sociedad, cambiar la sociedad. Actas del $8^{\circ}$ Congreso Interamericano de organización de instituciones educativas.

Sevilla, España: Secretariado de Publicaciones de la Universidad de Sevilla.

Castillo, S. (2002). Compromiso de la Evaluación Educativa. Madrid, España: Pearson Editorial S.A.

CEAAL. (2009). Biblioteca virtual de sistematización de experiencias. Recuperado el 10 de enero 2008 de http://www.alforja.or.cr/sistem/cgibin/blosxom.cgi/2009/05/19

Convenio Andrés Bello. (2004). Políticas, estrategias y consensos de acción en ciencias y tecnología de los países del Convenio Andrés Bello, 2003-2010. Vol.2. Colombia: Convenio Andrés Bello.

Estrada, V., Torres, L., Carvajal, A., Rodríguez, A. \& Bermúdez, C. (2005). Miradas sobre la sistematización de experiencias en trabajo social. Facultad de Humanidades. Escuela de Trabajo Social y Desarrollo Humano. Calí, Colombia: Universidad del Valle.

Federación para el aprendizaje. (2003). Los sistemas de aprendizaje de próxima generación y el papel de los maestros. En Visiones para el 2020: La transformación de la educación y el entrenamiento por medio de tecnologías avanzadas. EDUTEKA. Recuperado el 26 de marzo 2010 en http://www.eduteka.org/Visiones4.php 
Gestión en la Administración del Aprendizaje y Herramientas en la Sistematización de Experiencias

Greenberg, L. (2008). LMS and LCMS: What's the diference? Recuperado el 27 de marzo 2010 en www.ultimedia.co.uk/...Learning\%20and\%20LMS\%20Comparisons/LMS\%20an d\%20LCMS\%20Whats\%20the\%20Difference.doc

Gómez, D., Pérez, M. \& Curbelo, I. (2005). La gestión del conocimiento: Herramietna gerencial para las organizaciones de hoy. Revista AIBDA. (26), 1. San José, Costa Rica: IICA.

Guadamuz, L. (2006). La educación costarricense: Un problema y vigente...una solución pendiente. San José, Costa Rica: Impresiones técnicas de Costa Rica S.A.

Jara, H. O. (1994). Para sistematizar experiencias: Una propuesta teórica y práctica. San José, Costa Rica: Alforja.

lovanovich, L. (2007). Una propuesta metodológica para la Sistematización de la práctica docente en Educación de jóvenes y adultos. Revista Iberoamericana de Educación 42, 3, 25 marzo 2007. Organización de Estados Iberoamericanos para la Educación, la Ciencia y la Cultura (OEI). Recuperado el 7 de setiembre 2009 de http://www.rieoei.org/deloslectores/1897lovanovich.pdf

Messina, G. (2004). La sistematización educativa: Acerca de su especialidad. Revista Enfoques Educacionales. 6 (1): 19-28. Departamento de Educación, Facultad de Ciencias Sociales, Universidad de Chile. Recuperado el 28 de octubre 2008 de http://www.facso.uchile.cl/publicaciones/enfoques/index.html

Prat, E. \& Veyoda, D. (2008). Trece años de experiencias en Innovaciones Pedagógicas. Congreso Virtual Iberoamericano de Calidad en Educación a Distancia. EduQ@2008. Recuperado el 7 de setiembre 2009 en http://216.75.15.111/ joomlas/eduqa2008/images/ponencias/eje tematico 5/5 21 TRECE ANOS DE EXPERIENCIAS Prat Vedoya .pdf

Senlle, A. \& Gutierréz, N. (2005). Calidad en los Servicios Educativos. España: Editorial Díaz de Santos.

Watson, W. (2007). An Argument for Clarity: What are learning management systems. What are the not, and what should the become? Tech Trends. (51) 2.

Recuperado el 27 de marzo 2010 en http://www.springerlink.com/content/r1ur45g5t3225553/

Zapara, M. (2003). Sistemas de gestión del aprendizaje. Plataformas de teleformación. Recuperado el 20 de marzo 2010 en http://www.um.es/ead/red/9/SGA.pdf 